\title{
Analysis of the human thymic perivascular space during aging
}

\author{
Kristina G. Flores, ${ }^{1}$ Jie Li, ${ }^{1}$ Gregory D. Sempowski, ${ }^{2,3}$ Barton F. Haynes, ${ }^{2,3}$ \\ and Laura P. Hale ${ }^{1}$ \\ ${ }^{1}$ Department of Pathology, \\ ${ }^{2}$ Department of Medicine, and \\ ${ }^{3}$ Department of Immunology, Duke University Medical Center, Durham, North Carolina 27710, USA
}

Address correspondence to: Laura P. Hale, DUMC 2608, 185 Medical Sciences Research Building, Duke University Medical Center, Durham, North Carolina 27710, USA. Phone: (919) 684-4771; Fax: (919) 684-8756; E-mail: hale0004@mc.duke.edu.

Received for publication June 10, 1999, and accepted in revised form September 8, 1999.

\begin{abstract}
The perivascular space (PVS) of human thymus increases in volume during aging as thymopoiesis declines. Understanding the composition of the PVS is therefore vital to understanding mechanisms of thymic atrophy. We have analyzed 87 normal and 31 myasthenia gravis (MG) thymus tissues from patients ranging in age from newborn to 78 years, using immunohistologic and molecular assays. We confirmed that although thymic epithelial space (TES) volume decreases progressively with age, thymopoiesis with active T-cell receptor gene rearrangement continued normally within the TES into late life. Hematopoietic cells present in the adult PVS include T cells, B cells, and monocytes. Eosinophils are prominent in PVS of infants 2 years of age or younger. In the normal adult and the MG thymus, the PVS includes mature single-positive $\left(\mathrm{CD} 1 \mathrm{a}^{-}\right.$and $\mathrm{CD} 4^{+}$or $\left.\mathrm{CD} 8^{+}\right) \mathrm{T}$ lymphocytes that express CD45RO, and contains clusters of $\mathrm{T}$ cells expressing the TIA-1 cytotoxic granule antigen, suggesting a peripheral origin. PBMCs bind in vitro to MECA-79+ high endothelial venules present in the PVS, suggesting a mechanism for the recruitment of peripheral cells to thymic PVS. Therefore, in both normal subjects and MG patients, thymic PVS may be a compartment of the peripheral immune system that is not directly involved in thymopoiesis.
\end{abstract}

J. Clin. Invest. 104:1031-1039 (1999).

\section{Introduction}

The human thymic cortex contains densely packed immature thymocyte precursors within a thymic epithelial framework. In children, it is located directly beneath the thymic capsule. The thymic medulla is distinguished histologically by its less dense arrangement of thymocytes and by the presence of concentric whorls of keratin and terminally differentiated thymic epithelial cells called Hassall's bodies. The thymic cortex and medulla together comprise the thymic epithelial space (TES), where thymopoiesis occurs. Recent studies have focused attention on a third anatomic region of the thymus, known as the perivascular space (PVS) $(1,2)$ for its location adjacent to the blood vessels. The PVS is located within the thymic capsule, but is separated from the TES by a basement membrane and does not contain developing thymocytes $(3,4,5)$. Early in life, the thymus is composed primarily of TES that supports thymopoiesis and generation of the peripheral T-cell repertoire. As the thymus ages, the TES begins to atrophy, and the PVS increases in size as it fills with adipose tissue and lymphoid cells (2). However, the origin of cells within the thymic PVS and the role of the thymic PVS in the process of thymic atrophy are unclear. Hartwig and Steinmann have postulated that cytotoxic T lymphocytes (CTLs) migrating back to the thymus target medullary thymic epithelial cells expressing mutated self peptides that accumulate over time, thus inducing loss of thymic stroma and leading to thymic atrophy (6). For this hypothesis to be plausible, mature CTLs should be identifiable within the thymic medulla of normal thymus tissue undergoing age-related atrophy.

In this paper, we study the PVS in aging normal and myasthenia gravis (MG) human thymuses. We describe age-related changes in PVS cellular composition and demonstrate the mature phenotype of the T cells present in the PVS, including a population of cytotoxic granule-containing cells with the phenotype of mature CTLs.

\section{Methods}

Cells and tissues. Human thymus tissues were obtained as tissue discarded at the time of necessary cardiothoracic surgery or after thymectomy for the treatment of MG, according to a protocol approved by the Duke University Institutional Review Board for the use of discarded tissues. Tissues with thymoma were excluded. Portions of each tissue were fixed in $10 \%$ neutral buffered formalin for 6-24 hours, then processed into paraffin blocks using standard histologic procedures. Additional portions of most tissues were snap-frozen and stored at or below $-80^{\circ} \mathrm{C}$ until use. Formalin-fixed and paraffinembedded (FFPE) sections were used whenever possible to facilitate good histologic sampling of all tissue compartments. Thymus tissues were also obtained from the archives of the Duke University Department of Pathology as FFPE sections. All tissues were used anonymously, with recording of only patient age, gender, and surgical diagnosis. Table 1 describes the clinical characteristics of the patients from whom thymic tissue was obtained. PBMCs for high endothelial venule (HEV) binding assays 
were obtained from human blood by density gradient centrifugation with Lymphocyte Separation Medium (ICN Biomedicals Inc., Costa Mesa, California, USA).

Antibodies. The following monoclonal and polyclonal antibodies were used: anti-cytokeratin (cocktail of AE1/AE3 from Boehringer Mannheim Biochemicals Inc., Indianapolis, Indiana, USA and CAM5.2 from Becton Dickinson Immunocytometry Systems, San Jose, California, USA); CD1a (O10; kind gift of L. Boumsell), INSERM, Creteil, France) (7); CD3 (rabbit polyclonal A0452; DAKO Corp., Carpinteria, California, USA) (8); CD4 (1F6; Novocastra Laboratories Ltd., Newcastle upon Tyne, United Kingdom); CD8 (C8/144B; DAKO Corp.); CD20 (L26; DAKO Corp.); CD38 (T16; Beckman Coulter Inc., Miami, Florida, USA); AT13/5 (Harlan Bioproducts for Science Inc., Indianapolis, Indiana, USA); CD45RA (HI115; obtained through the 6th International Workshop on Leukocyte Differentiation Antigens); CD45RO (UCHL-1; American Type Culture Collection, Rockville, Maryland, USA); CD68 (KP-1; DAKO Corp.); Ki-67 nuclear proliferation antigen (mib-1; Beckman Coulter) (9); anti-cytotoxic granule protein (TIA-1; Beckman Coulter Inc.) (10); anti-PVS (TE-7) (11); and anti-HEV (MECA-79 and MECA-367; kind gifts of E. Butcher, Stanford University, Stanford, California, USA) $(12,13)$. Isotype-matched control antibodies were obtained from Sigma Chemical Co. (St. Louis, Missouri, USA) and Southern Biotechnology Associates (Birmingham, Alabama, USA), or were generated locally. CD4FITC and CD8-PC5 directly conjugated fluorescent antibodies were obtained from PharMingen (San Diego, California, USA). CD3-FITC and CD38-PE mAb's were obtained from Beckman Coulter Inc.

Immunohistologic studies. Immunohistochemical staining was performed on 4- $\mu \mathrm{m}$ FFPE sections using standard protocols, including blocking of endogenous peroxidase activity $\left(0.6 \% \mathrm{H}_{2} \mathrm{O}_{2}\right.$ in absolute methanol for 15 minutes), microwave citrate antigen retrieval (BioGenex Laboratories, San Ramon, California, USA), and blocking with diluted goat or horse serum and avidin-biotin (Vector Laboratories, Burlingame, California, USA). The slides were then sequentially incubated at $37^{\circ} \mathrm{C}$ with primary antibody, biotinylated secondary antibody, and avidin-biotin horseradish peroxidase complexes (VECTASTAIN ABC; Vector Laboratories), with intervening PBS washes. Bound antibody was detected with 3,3'diaminobenzidine and $\mathrm{H}_{2} \mathrm{O}_{2}$. Double immunostains also used alkaline phosphatase (VECTASTAIN ABC-AP) and Vector Red phosphatase substrate (Vector Laboratories). Frozen sections were stained as described after acetone fixation, with omission of deparaffinization, antigen retrieval, and blocking steps. For multicolor immunofluorescence staining, frozen sections were first reacted with cytokeratin $\mathrm{mAb}$ 's in immunoperoxidase assays to locate the TES. They were then incubated further with fluorophore-conjugated antibodies for $30 \mathrm{~min}$ utes at room temperature, washed, and then examined using an Olympus AX70 microscope equipped for multicolor fluorescence and computerized image analysis.

The percentage of thymus area consisting of PVS was determined by microscopic examination of hematoxylin and eosin-stained (H\&E-stained) and cytokeratin- immunostained sections, using a Zeiss video microscope with grid overlay. Nonlymphoid components of the PVS can also be stained directly with mAb TE7 (11); however, this mAb works only on frozen sections (data not shown). PVS percentages were obtained for at least 6 representative $10 \times\left(1.9 \mathrm{~mm}^{2}\right)$ fields, and then averaged to obtain the mean. The correlation between percent PVS obtained using cytokeratin-immunostained sections compared with H\&E-stained sections was 0.97.

For quantitation of cell types present in TES and PVS regions, immunostained sections were classified according to the following criteria, using a Zeiss video microscope with grid overlay: $0=$ rare to absent positive cells; $1+=$ less than $5 \%$ positive cells; $2+=5-30 \%$ positive cells; $3+=31-80 \%$ positive cells; and $4+=$ more than $80 \%$ positive cells.

Flow cytometric studies. Lymphocytes obtained from pediatric and adult thymus were reacted with combinations of directly conjugated fluorescent antibodies for 30 minutes at $4^{\circ} \mathrm{C}$, washed with PBS plus $1 \%$ BSA, fixed with $0.4 \%$ paraformaldehyde, and analyzed on a FACStar Plus flow cytometer (Becton Dickinson Immunocytometry Systems) at the Duke University Center for AIDS Research Flow Cytometry Facility.

Ligation-mediated PCR assay. Genomic DNA was isolated from thymocytes as described previously, taking care not to induce random DNA cleavage during the isolation procedure $(14,15)$. DNA was resuspended in $10 \mathrm{mM}$ Tris and $1 \mathrm{mM}$ EDTA, and then stored at $4^{\circ} \mathrm{C}$ until assayed.

Ligation-mediated PCR (LM-PCR) to detect doublestranded DNA (dsDNA) breaks was modified from previously described protocols (14) and optimized for use with human DNA. Breaks occurring $5^{\prime}$ or $3^{\prime}$ to the Tcell receptor $\beta$ chain (TCRB) D $\beta 2.1$ locus were analyzed to achieve a maximal 50\% detection of V-DJ and D-J breaks occurring at this locus, which is 1 of 2 human $D \beta$ loci. A double-stranded asymmetrical linker was generated as described (14). Genomic DNA (1-2 $\mu \mathrm{g})$ was ligated to $20 \mathrm{pmol}$ of asymmetrical linker for 12-16 hours at $16^{\circ} \mathrm{C}$. The reaction was stopped by the addition of 1 volume of $10 \mathrm{mM}$ Tris, $50 \mathrm{mM} \mathrm{KCl}$, and $0.1 \%$ Triton- $X 100$, and then heating at $95^{\circ} \mathrm{C}$ for 15 minutes. Linker-ligated DNA was stored at $-20^{\circ} \mathrm{C}$.

Serial 8-fold dilutions of linker-ligated human thymus DNA were made into mock linker-ligated mouse DNA to keep the total amount of DNA constant for each sample. Breaks in dsDNA were then detected by nested PCR using a linker-specific primer and primers that flank the $\mathrm{D} \beta 2.1$ locus: linker 2 primer: 5'-CCGGGAGATCTGAATTCCAC-3'; outer forward primer: 5'-GGAGTTCAGACATCGTTCAG-3'; outer reverse primer: $5^{\prime}$-CCACCAATTTGCAGAGGAGA3'; inner forward primer: 5'-CTGGATTGTTTGTCCTCCTG3'; and inner reverse primer: 5'-GGGACAACACAGTGGATTTG- $3^{\prime}$. Nested PCR $\left(60\right.$ seconds at $94^{\circ} \mathrm{C}, 30$ seconds at $94^{\circ} \mathrm{C}, 30$ seconds at $62^{\circ} \mathrm{C}, 45$ seconds at $72^{\circ} \mathrm{C}$, and a final 8-minute extension step at $72^{\circ} \mathrm{C}$ ) was performed in $37-\mu \mathrm{L}$ reactions in hot-start PCR tubes or by heated-lid PCR. First-round PCR reactions contained $20 \mu \mathrm{M}$ each of linker 2 primer and forward outer or reverse outer primer, along with 219 ng of starting DNA. First-round PCR reactions were carried out for 15 cycles. Two microliters of each reaction preparation was carried into secondround PCR containing $20 \mu \mathrm{M}$ each of linker 2 and for- 
ward inner or reverse inner primer for 30 cycles. Template-control PCR reactions using forward outer and reverse outer primers only were carried out for 35 cycles. PCR products were analyzed on a $2 \%$ agarose gel, blotted according to standard procedures, and then detected with a ${ }^{32} \mathrm{P}$-labeled cDNA probe corresponding to $\mathrm{bp}$ 277-943 of the TCRB locus (16).

Lymphocyte binding assay. The adherence of human PBMCs to HEVs in the thymic PVS was performed as described $(17,18)$. Frozen thymus or control tissue sections $(12 \mu \mathrm{m})$ were overlaid with $200 \mu \mathrm{L}$ of PBMCs $(15 \times$ $10^{6}$ cells $\left./ \mathrm{mL}\right)$ and incubated at $4{ }^{\circ} \mathrm{C}$ with rotation $(64$ $\mathrm{rpm}$ ) for 30 minutes. The cell suspension was gently decanted, and the slides were fixed in $2.4 \%$ glutaraldehyde in PBS overnight at $4^{\circ} \mathrm{C}$. Slides were then washed twice with PBS, counterstained with hematoxylin, and mounted with glycerol gelatin (Sigma Chemical Co.). The number of PBMCs adherent to each HEV was determined by direct microscopic visualization of coded slides by a blinded observer. The total number and location of HEVs in each section was confirmed by MECA-79 immunostaining of an adjacent tissue section.

$R$ Nase protection assays. Total RNA was isolated from approximately $100 \mathrm{mg}$ of tissue by homogenization with a tissue disrupter (OMNI International Inc., Marietta, Georgia, USA) fitted with disposable 7-mm tips, in $1 \mathrm{~mL}$ of Trizol (GIBCO BRL, Gaithersburg, Maryland, USA) followed by extraction per the manufacturer's protocol. Total RNA concentration was determined by measuring absorbance at $260 \mathrm{~nm}$. Steady-state levels of specific cytokine mRNAs in tissues were determined using the RiboQuant Multi-Probe RNase Protection Assay System (PharMingen). Ten micrograms of RNA of each tissue sample was hybridized overnight with ${ }^{32} \mathrm{P}$-labeled riboprobe sets, and then processed as described in the manufacturer's instructions. Protected RNA transcripts were separated on a $5 \%$ denaturing polyacrylamide gel. Cytokine mRNA bands were quantified by PhosphorImager analysis (Molecular Dynamics, Sunnyvale, California, USA) and reported as percent of GAPDH signal.

\section{Results}

Thymic PVS volume increases with age in normal subjects and in MG patients. Although grossly detectable thymic atrophy may not occur until puberty, analysis of thymic PVS in normal thymus tissues ranging in age from 0 to 78 years (Table 1 ) clearly demonstrates that a statistically significant increase in the PVS has occurred by $2-10$ years of age (age quintile $2 ; P<0.01$ vs. age quintile 1 [0-1 year]) (Figure $1 \mathrm{a})$. The percent PVS increases progressively with age in normal individuals (Figure 1a), in agreement with previous studies by Steinmann (2). The increase in PVS volume is accompanied by accumulation of lymphocytes within the PVS (Figure 1, c-h), particularly in quintiles $2-4$.

The contribution of the TES and the PVS to the overall composition of MG

Table 1 thymus during aging was determined and compared with normal thymus. The percent PVS is increased in MG thymuses compared with age-matched normal thymuses (quintile 2, $P<0.07$; quintile $3, P<0.08$; quintile $4, P<0.0006$; quintile $5, P<0.018$ ) (Figure $1 b$ ). A portion of this increase is due to follicular hyperplasia present in a subset of MG thymus tissue (Table 1). Cytokeratin staining (Figure 1h) clearly demonstrates that the primary and secondary follicles present in MG thymus with follicular hyperplasia occur in the PVS, not in the TES, similar to what is observed in normal aging thymus (5). Therefore, the thymic follicular hyperplasia seen in the PVS of MG thymus is an exaggerated state of the perivascular infiltration with lymphocytes that normally occurs in aging thymus.

The adipocyte and lymphocyte content of the PVS similarly changes with age. Adipocytes are rare in quintile 1, and increase progressively in quintiles $2-5$, as shown in Figure 1 . The lymphocyte content of the PVS increases rapidly in quintiles 2 and 3; a significant fraction of PVS area in these age ranges contains lymphocytes (Figure 1, $d$ and e), as reported previously by Steinmann (2). The lymphocyte content of the PVS begins to decrease in quintile 4 , with a corresponding increase in the fraction of PVS composed of adipose tissue in quintiles 4 and 5 , relative to earlier quintiles (Figure 1, f and g). The PVS of most tissue samples in quintile 5 contains predominately adipocytes, with few lymphocytes (Figure 1g).

Immunohistologic identification of cells undergoing thymopoiesis in the TES. The TES in both normal and MG thymus decreases with age as PVS increases (Figure 1, a and b). To study age-related changes in thymopoiesis, we have determined the phenotype of lymphocytes present in the TES of normal and MG thymus tissues. CD1a is expressed on immature $\mathrm{CD}^{+}, \mathrm{CD}^{+}$(double-positive) cortical thymocytes that have successfully completed rearrangement of the TCR $\beta$-chain gene locus (19), but it is not expressed on medullary thymocytes or mature T cells (20). We found that CD1a immunoreactivity in infant thymus (age $0-1$ year) was limited to thymocytes within the thymic cortex (data not shown). Some dendritic cells located in the thymic medulla also reacted with CD1a mAb; however, these cells were clearly distinguished from immature thymocytes by their location, larger size, and multiple dendritic processes. Rare CD1 ${ }^{+}$cells with dendritic cell morphology were also

Characteristics of studied thymus tissues ${ }^{\mathrm{A}}$

\begin{tabular}{lccccc} 
& \multicolumn{5}{c}{ Age quintile $^{\mathrm{A}}$} \\
Diagnosis & 1 & 2 & 3 & 4 & 5 \\
Cardiothoracic surgery & $18(10 / 7 / 1)^{\mathrm{B}}$ & $17(10 / 7)$ & $5(2 / 3)$ & $8(3 / 5)$ & $7(5 / 2)$ \\
Mediastinal mass & 0 & $1(0 / 1)$ & 0 & $2(1 / 1)$ & 0 \\
Thyroid/Parathyroid & 0 & $1(0 / 1)$ & $5(2 / 3)$ & $8(2 / 6)$ & $12(0 / 12)$ \\
Trauma & 0 & 0 & 0 & $3(3 / 0)$ & 0 \\
Normal tissues & $18(10 / 7 / 1)$ & $19(10 / 9)$ & $10(4 / 6)$ & $21(9 / 12)$ & $19(5 / 14)$ \\
$\quad$ Total normal tissues 87 (38/48/1) & & $2(0 / 2)$ & $8(2 / 6)$ & $13(5 / 8)$ & $8(6 / 2)$ \\
MG & 0 & $50 \%$ & $63 \%$ & $31 \%$ & $32 \%$ \\
Percent with follicular hyperplasia & & & & & \\
Total MG tissues 31 (13/18) & & & &
\end{tabular}

Total MG tissues $31(13 / 18)$

${ }^{A}$ Data are expressed as total number of tissues (males/females) in each diagnosis category. Age quintiles are defined as follows: $1=0-1$ year; $2=2-10$ years; $3=11-25$ years; $4=26-49$ years; $5=50$ years or older. ${ }^{B} \mathrm{ln}$ age quintile 1 , the gender of 1 thymus donor was not recorded. 

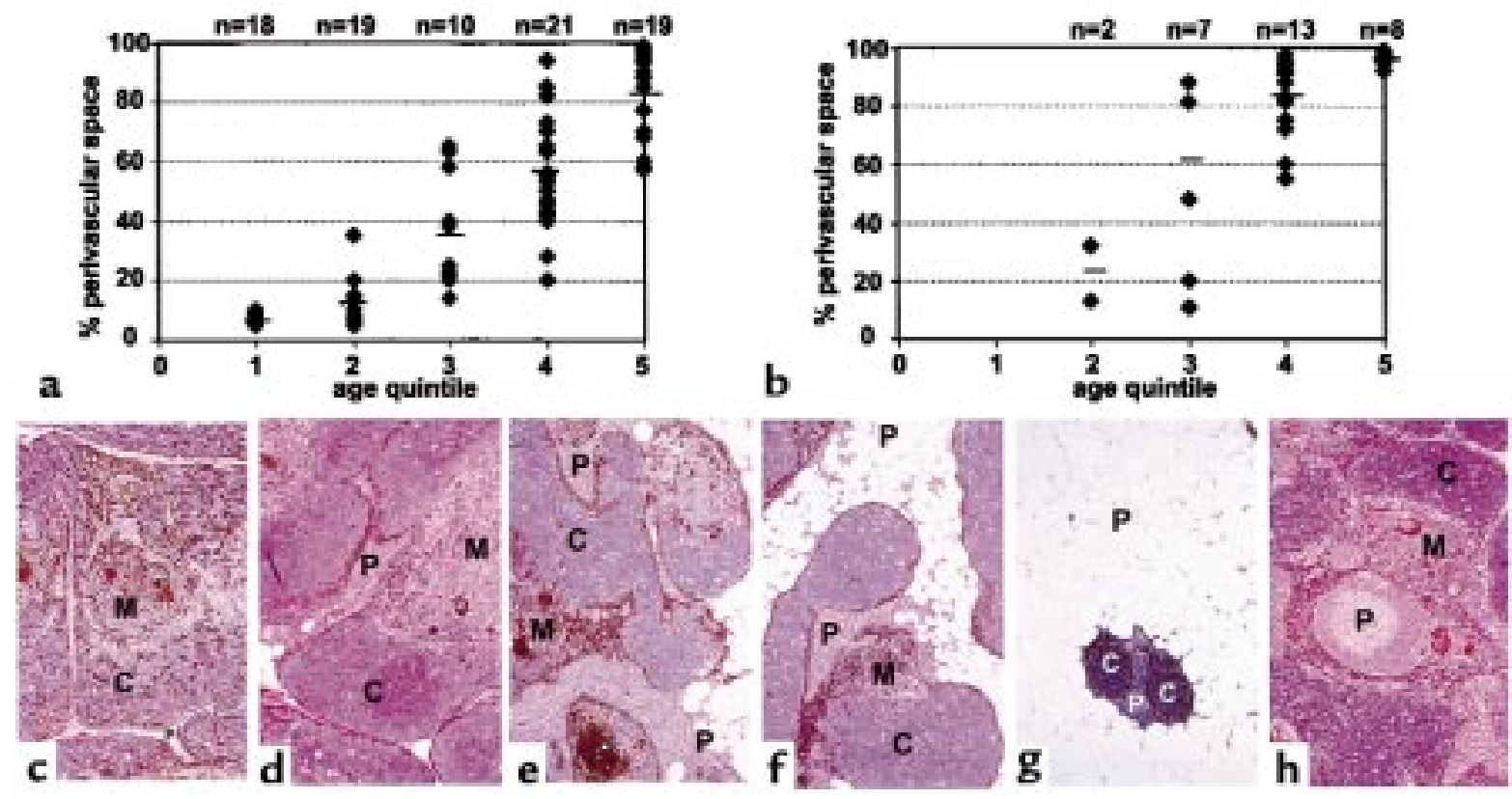

Figure 1

Thymic PVS increases with age in normal individuals and in patients with MG. The percent thymic PVS determined from H\&E-stained sections is shown as a function of age quintile. (a) Data for normal individuals are expressed as mean \pm SD for the indicated number of cases. Quintile 1: $7 \pm$ $2 \%, n=18$; quintile 2: $12 \pm 7 \%, n=19$; quintile 3: $37 \pm 19 \%, n=10$; quintile $4: 55 \pm 18 \%, n=21$; quintile $5: 82 \pm 16 \%, n=19$.(b) Data for patients with MG are expressed as mean \pm SD for the indicated number of cases. Quintile 2: $23 \pm 13 \%, n=2$; quintile 3: $61 \pm 34 \%, n=8$; quintile 4: $83 \pm 14 \%$, $n=13$; quintile 5: $96 \pm 2 \%, n=8$. ( $\mathbf{c}-\mathbf{h})$ Cytokeratin immunoperoxidase staining (brown color) outlines TES, with an H\&E counterstain. Letters denote representative regions of thymic cortex (C), medulla (M), and PVS (P). (c) Quintile 1. (d) Quintile 2. (e) Quintile 3. (f) Quintile 4. (g) Quintile 5. (h) Thymus with follicular hyperplasia from a 20-year-old female with MG. Cytokeratin and H\&E staining shows that primary and secondary follicles are located outside the cytokeratin network, within the PVS. $\times 25$ (original magnification).

present in the PVS. The majority of the $\mathrm{CD} 1 \mathrm{a}^{+}$cortical thymocytes also reacted with mib-1 mAb specific for the Ki-67 nuclear proliferation antigen, with less frequent mib- $1^{+}$cells present in the thymic medulla and in the PVS (not shown). $\mathrm{CD} \mathrm{a}^{+}$, mib- $1^{+}$cortical thymocytes also reacted strongly with antibodies specific for CD3, CD4, CD8, CD38, and CD45RO (not shown), as expected for immature thymocytes $(20,21)$. Only rare isolated lymphocytes within the TES were reactive with TIA- $1 \mathrm{mAb}$, specific for granules present in activated CTLs. Cytokeratin staining revealed a loose network of thymic epithelial (TE) cells within the cortex in association with large numbers of CD1a $\mathrm{a}^{+}$, mib-1 $1^{+}$thymocytes, with a somewhat denser pattern of TE cells in the medulla (not shown). The majority of TE cells in both cortex and medulla were surrounded by multiple thymocytes. Taken together, these studies suggest that thymus tissues that are active in the process of thymopoiesis can be identified immunohistologically in FFPE tissues by the presence of immature $\mathrm{CD} 1 \mathrm{a}^{+}$, mib-1 $1^{+}$thymocytes in a loose network of TE cells that allows thymocyte-TE cell interactions.

Of the 118 normal and MG thymus tissues examined, 116 contained at least some regions meeting our immunohistologic criteria for thymopoiesis. However, the absolute numbers of immature CD1a+, mib-1+ thymocytes undergoing thymopoiesis decreased progressively as the percent TES decreased with age and in MG. Thymocytes derived from a 78-year-old male thymus shown by immunohistochemistry to contain $\mathrm{CD} 1 \mathrm{a}^{+}$, mib-1 $1^{+}$thymocytes displayed an immature phenotype.
These thymocytes were $\mathrm{CD}^{+}$and $\mathrm{CD}^{+}$, expressed CD45RO and CD38, and were negative for CD95, CD62L, and CD45RA (Figure 2). This thymus was highly atrophic with few PVS lymphocytes present. Although the absolute numbers of immature thymocytes were greatly decreased, the relative proportions were similar to those in pediatric thymus.

$L M-P C R$ detects ongoing TCR gene rearrangement in pediatric and adult human thymocytes. To provide molecular confirmation of thymopoiesis, we used an LM-PCR assay $(22,14)$ to identify dsDNA breaks indicative of D-J or VDJ TCR gene rearrangement in thymocytes derived from pediatric and adult thymus tissues. This assay detects dsDNA breaks that are transiently generated at recombination signal sequences by the V-D-J recombinase complex during the process of TCR gene rearrangement (Figure 3a). The presence of LM-PCR signals in a given tissue is thus indicative of ongoing thymopoiesis.

LM-PCR analysis detected DNA breaks associated with both D-J and V-DJ rearrangement of the TCRB gene in 14 of 14 pediatric thymocyte samples tested (2 representative samples are shown in Figure 3b, lanes 1, 3, 9, and 11). This assay is specific for ongoing human TCRB gene rearrangement: no LM-PCR signals are detected in DNA from human spleen or tonsil, mouse thymocytes (data not shown), bacteria (Figure 3b, lanes 5, 6, 13, and 14), or mock linker-ligated thymocytes (Figure 3b, lanes 2, 4, 10, and 12).

LM-PCR was used to determine whether the phenotypically immature thymocytes present in older adult 
thymuses were undergoing thymopoiesis. LM-PCR analysis detected dsDNA breaks corresponding to both D-J and V-DJ TCR gene rearrangement in most thymocyte DNA samples derived from normal adult and MG thymus tissues that showed immunohistologic evidence of thymopoiesis (Figure 3c). LM-PCR signals corresponding to D-J TCR gene rearrangement were detected in 8 of 8 adult thymus tissues tested, with V-DJ signals detected in 5 of 8 samples.

Eosinophils are prominent in the PVS of pediatric thymus. The PVS of infant thymus (quintile $1 ; 0-1$ year) contains few lymphocytes. The majority of the cells present in the PVS of infants are spindle-shaped, consistent with fibroblasts or preadipocytes. However, large clusters of eosinophils are also frequently found in the PVS of infant thymus (Figure 4a). Many of these eosinophils have the bilobed nucleus characteristic of mature eosinophils; also present are cells containing similar eosinophilic granules and large single-lobed or indented nuclei with chromatin patterns that are consistent with the eosinophilic myelocyte and eosinophilic metamyelocyte stages of eosinophil differentiation. Clusters of eosinophils are smaller and less abundant in older children (quintile 2; $2-10$ years). Eosinophils are rare to nondetectable in older thymus tissues from age quintiles 3-5 (11 years or older).

The eosinophils present within the PVS of pediatric thymus could have differentiated there in situ or could have developed inside the TES or outside the thymus, and then migrated into the PVS. To investigate the ability of the thymus to support the differentiation of eosinophils, we analyzed thymic IL-5 mRNA production as a function of age. In humans, IL-5 is highly specific for stimulating the production, activation, and survival of eosinophils (23). In mice, the expression of IL-5 is sufficient to induce the full pathway of eosinophil differentiation (24). Therefore, we analyzed the expression of IL-5 in 44 normal human thymus tissues by RNase protection assay. We found that IL-5 was readily detectable in most normal thymus tissues from patients 2 years of age or younger (IL- $5=1.13 \pm 0.28 \%$ of GAPDH signal, range $0-3.28, n=14$ ). IL-5 mRNA was unde- tectable in thymus derived from patients 3 years of age or older $(n=30)$. The level of IL-5 mRNA correlates with the observed incidence of eosinophilia within the thymic PVS in patients 3 years of age or younger. In particular, thymus tissues with high IL-5 content had moderate to large clusters of eosinophils present in the PVS (Figure 4a). Conversely, thymic tissues with undetectable IL-5 mRNA levels did not demonstrate eosinophilia of the thymic PVS. Taken together with the observation that some of the eosinophils present in the PVS exhibit nuclear morphologies characteristic of immature eosinophils, these data suggest that the eosinophils present in the thymic PVS of infants could differentiate in the PVS in response to thymic IL-5 production.

PVS lymphocytes have a phenotype consistent with peripheral lymphocytes. As described above, T lymphocytes present within the PVS are not positive for both CD1a and mib1 and are therefore most likely mature T lymphocytes. Mature T lymphocytes present in the PVS may be newly generated mature virgin $T$ cells emigrating from the thymus or T cells recirculating from the periphery. No phenotypic markers currently exist to specifically detect recent thymic emigrants in humans. Therefore, we determined the immunoreactivity of PVS lymphocytes with a panel of antibodies directed at lymphocyte differentiation and activation markers, using single, double, and triple immunohistochemical and immunofluorescence assays to locate the PVS (by lack of cytokeratin reactivity; Figure 5, a and b) and to identify appropriate markers on lymphocytes. Results are summarized in Table 2.

When large numbers of lymphocytes are present in the PVS of normal thymus (usually in age quintiles 3 and 4), the infiltrate consists of both T cells and B cells (Figure 5, $\mathrm{c}$ and $\mathrm{d})$. $\mathrm{T}$ cells in the PVS are CD1a $\mathrm{a}^{-}$and most are also mib-1- (Figure 5, e and f). Triple immunostains, combining a cytokeratin immunoperoxidase stain to view the TES with CD4-FITC and CD8-PC5 fluorescent antibodies, demonstrated that PVS T cells are single-positive, expressing either CD4 or CD8, but not both (not shown; see also Figure 5g). Most PVS T cells express the CD45RO surface antigen (Figure $5 \mathrm{~h}$ ) rather than the CD45RA antigen typi-

\section{Figure 2}

Phenotypically immature thymocytes are present in adult thymus tissues meeting immunohistologic criteria for thymopoiesis. Lymphocytes from a 78-yearold male thymus were analyzed by flow cytometry using combinations of fluorescently labeled mAb's. The majority of lymphocytes present were $\mathrm{CD}^{+}(\mathbf{a})$, with $82 \% \mathrm{CD}^{+}, \mathrm{CD}^{+}$double-positive (b). $3 \%$ of cells were reactive with CD19 and CD20 mAb's (c), and were consequently identified as B lymphocytes. Of the CD4hi cells, more than $90 \%$ were CD45RO+ (d), $97 \%$ were $\mathrm{CD}^{3} 8^{+}(\mathbf{e})$, and $97 \%$ were CD45RA(f), consistent with an immature phenotype. Similar results were seen with gating on $\mathrm{CD}^{+}$lymphocytes. Very few mature T cells were present in this sample, consistent with the observed lack of lymphocytes infiltrating the PVS on immunohistochemical sections. Although the majority of cells present in this thymus were immature thymocytes, the absolute numbers of thymocytes obtained for analysis were less than $1 \%$ of those obtained per gram of tissue from pediatric thymus.
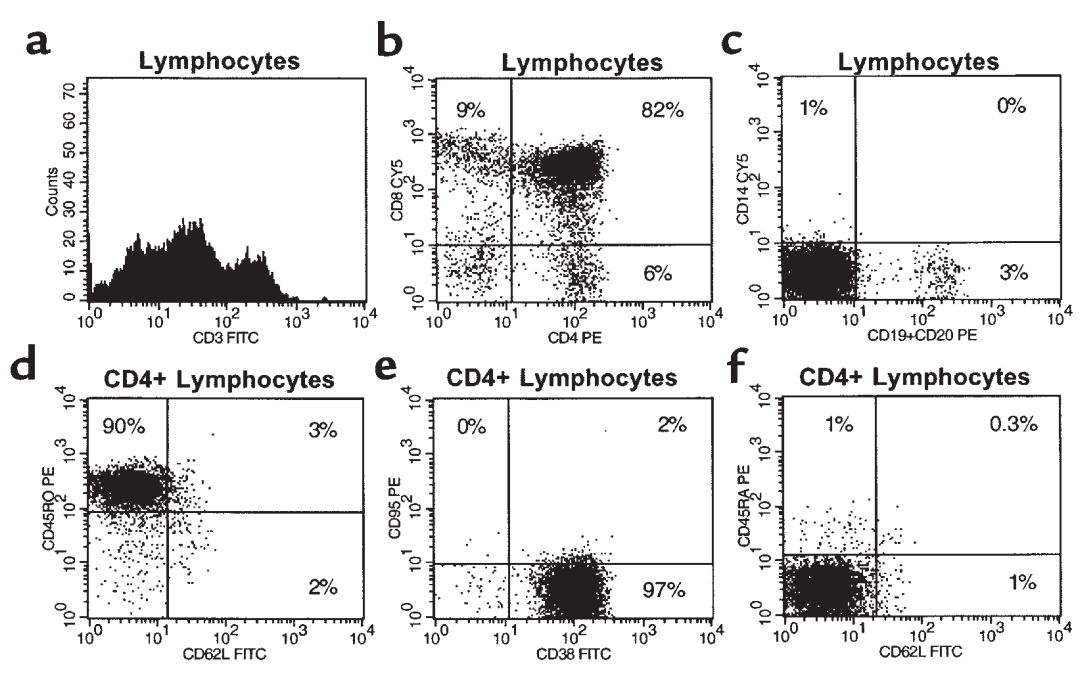
cally expressed on peripheral virgin T cells (not shown). Although large numbers of CD45RA ${ }^{+}$lymphocytes are present both in the thymic medulla and in the PVS, the majority of these cells are B cells (Figure $4 \mathrm{~b}$ ). The cell types present in the PVS of 50 normal adult and 31 age-matched MG thymus tissues are similar, with further expansion of the PVS in MG thymus with follicular hyperplasia by infiltrates of primarily B lymphocytes, often with prominent germinal centers. (Figure $1 \mathrm{~h}$ ).

We observed that a subset of the $\mathrm{CD}^{+} \mathrm{T}$ cells present in the PVS also express the TIA-1 antigen characteristic of activated CTLs (Figure 4c), indicating that these cells are not recent thymic emigrants, but are mature CTLs from the periphery (25). We also found differences in CD38 expression between T cells in the TES and the PVS. Although cortical and medullary thymocytes express relatively high reactivity with CD38 mAb (21) (Figure 2e), most T cells present in the PVS do not have high reactivity with CD38. However, germinal center $\mathrm{B}$

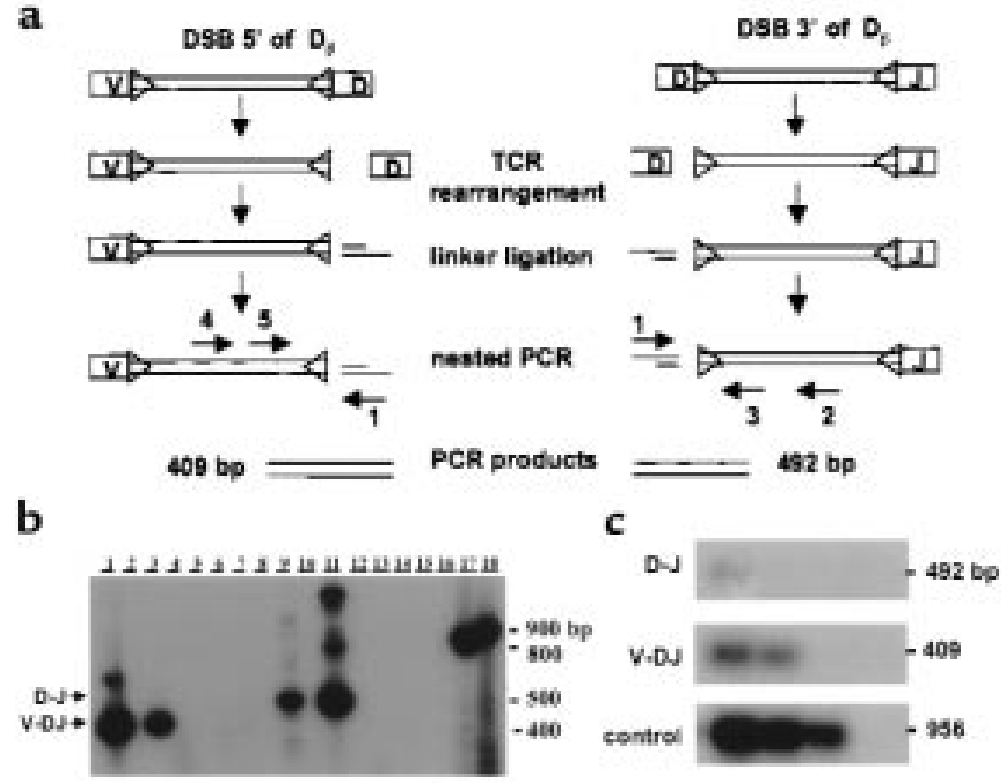

Figure 3

LM-PCR detects ongoing TCR gene rearrangement in pediatric and adult thymocytes. (a) LM-PCR detects free signal ends generated by dsDNA breaks $3^{\prime}$ and $5^{\prime}$ of the D $\beta 2.1$ TCR gene segment, corresponding to D-J and V-DJ rearrangements, respectively. (b) Specific LM-PCR products obtained from thymocytes from 2 normal individuals less than 6 months old, indicating ongoing V-DJ (lanes 1 and 3; 409 bp) and D-J (lanes 9 and 11; 492 bp) rearrangement. Controls with non-linker-ligated DNA amplified with primers 3 and 5 (lane 17) or 2 and 4 (lane 18) demonstrate the appropriately sized germline bands (868 and 956 bp, respectively). Lanes using mock-ligated DNA (lanes $2,4,10$, and 12), DNA lacking the TCR loci (bacterial DNA \pm linker ligation; lanes 5, 6, 13, and 14), linker alone (lanes 7 and 15), and PCR blanks (lanes 8 and 16) are negative. The higher molecular weight bands seen in lanes 1 and 11 probably represent dsDNA breaks corresponding to additional (nonproductive) rearrangements in cells with a rearranged $D \beta 2.1$ locus. However, this remains to be formally demonstrated using probes and primers specific for sequences unique to these downstream regions. (c) LM-PCR signals generated from thymocytes obtained from a 24-year-old male. Eight-fold dilutions of DNA (decreasing concentration left to right) were linker ligated and subjected to LM-PCR as described. Signals corresponding to both D-J and V-DJ rearrangements were detected in 5 of 8 samples (donor age and gender: $24 \mathrm{M}, 29 \mathrm{~F}, 27 \mathrm{~F}$, $41 \mathrm{~F}, 42 \mathrm{~F}$ ). Only D-J signals were detected in 3 samples (donor age and gender: $28 \mathrm{~F}, 34 \mathrm{~F}$, $46 \mathrm{M}$ ). All tissues tested had immunohistologic evidence for thymopoiesis, with at least small foci of $\mathrm{CD} \mathrm{a}^{+}$, mib-1+ lymphocytes within a loose network of thymic epithelial cells. Template control reactions using primers 2 and 4 amplified the appropriately sized germline band. cells are moderately to strongly reactive with CD38 staining to locate the TES, combined with CD3-FITC (data not shown). Conversely, the majorwith $\mathrm{CD} 38 \mathrm{mAb}$. Less than $5 \%$ of PVS $\mathrm{CD}^{+}$cells reactstrongly with CD38 mAb, and these cells were freThenotype. The relative lack of CD38 immunostaining of most PVS T cells is also consistent with a peripheral ral blood lymphocytes are also CD38- (21).

PBMCs bind to MECA-79+ HEVs in the PVS of adult normal and $M G$ thymus. We observed that HEVs could frequently be identified in normal thymus PVS when large numbers fes were present in the PVS. Similarly, thymus from adult patients with MG that had large amounts of PVS lymphoid infiltrates also contained HEVs within the PVS. Peripheral lymph node HEVs have previously been shown to be immunoreactive with MECA$79 \mathrm{mAb}$, which recognizes a ligand for Lselectin (13). We found that the HEVs present in the PVS of adult normal and MG thymus react strongly with MECA-79 mAb (Figure 5i), similarly to HEVs in lymph nodes that have been shown to support lymphocyte recirculation through a mechanism that is dependent on MECA-79 and Lselectin (26). Thymic PVS HEVs did not react with mAb MECA-367 (not shown), which is specific for a mucosal addressin previously shown to direct migration of lymphocytes to Peyer's patches and mesenteric lymph nodes $(12,27,28)$.

To determine whether the thymic PVS HEVs can potentially support the emigration of peripheral lymphocytes, we performed in vitro assays of PBMC binding to tissue sections, as described previously (17, 18). We found that PBMCs bound specifically to thymic PVS HEVs, with $14 \pm 3$ lymphocytes (mean \pm SD) bound per HEV in the 4 thymus tissues tested. These studies demonstrate that PBMCs bind well to the MECA $-79^{+}$thymic HEVs, which can thus potentially direct the migration of peripheral lymphocytes into the thymic PVS. Additional studies will be needed to determine the precise mechanisms for lymphocyte emigration into the PVS.

\section{Discussion}

In this paper, we have extensively characterized the thymic PVS of adults and provided data suggesting a peripheral origin for PVS lymphocytes. Our identification of MECA$79^{+} \mathrm{HEVs}$ that are capable of binding PBMCs suggests a mechanism for egress of peripher- 

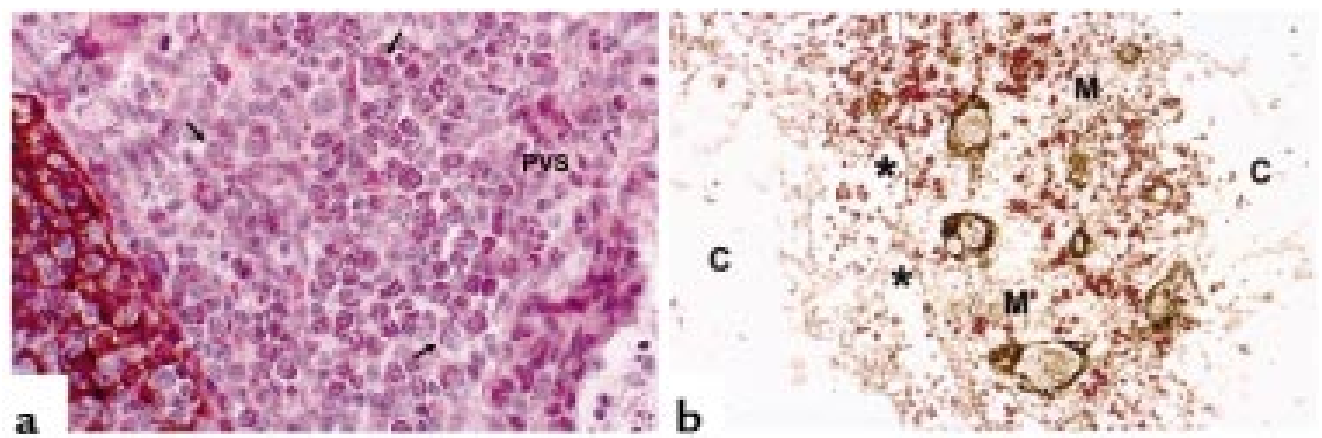

Figure 4

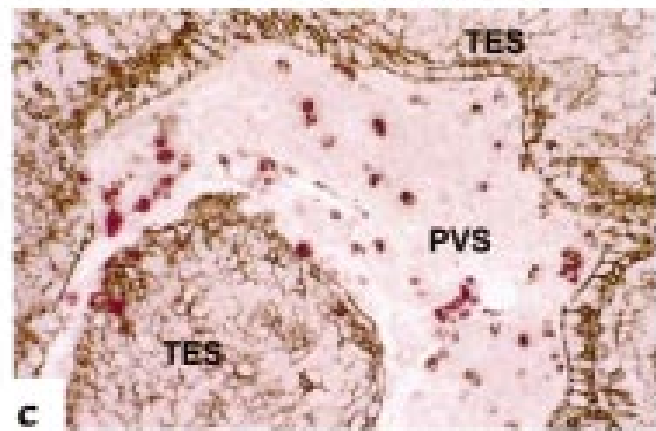

(C). (c) Cytokeratin (brown) and TIA-1 (red) double staining identifies TIA $-1^{+}$cells within the PVS.

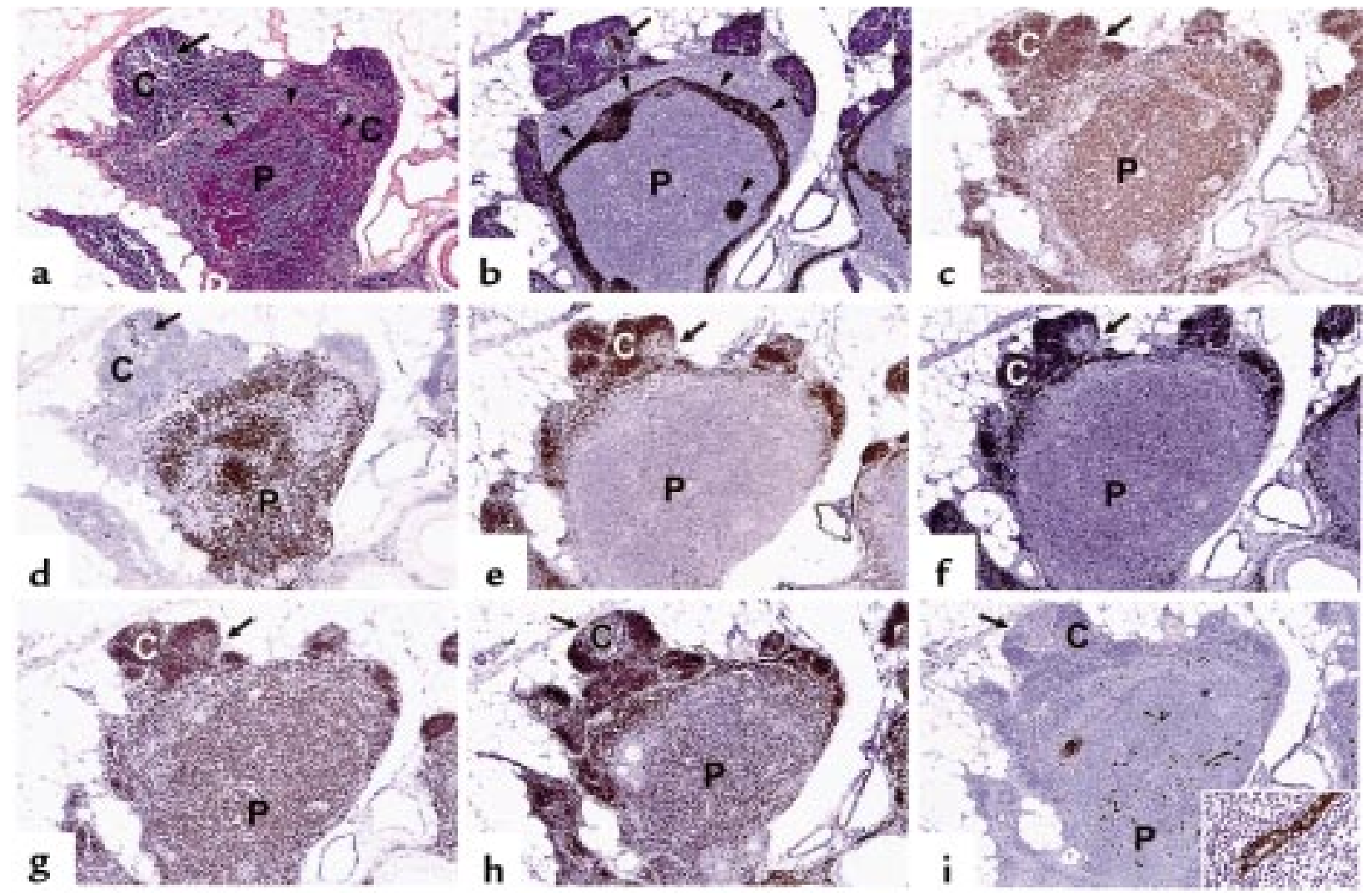

Figure 5

Phenotypes of cells present in the thymic PVS. Thymus from a 42-year-old female with MG is shown in order to allow examination of relatively large areas of PVS within a single field. TES active in thymopoiesis is highlighted using a $C$ to indicate active cortex, with an arrow pointing to the medulla. The cytokeratin stain (b) also demonstrates inactive TES (arrowheads in $\mathbf{a}$ and $\mathbf{b}$ ) surrounded by PVS (P). The following immunoperoxidase stains demonstrate phenotypes of cells present in both TES and PVS: (c) CD3, T cells; (d) CD20, B cells; (e) CD1a, immature thymocytes; (f) mib-1, proliferating cells (note the positive reaction of both CD1a and mib-1 mAb's [brown] with thymocytes in cortex [C] but not in medulla [arrow]); (g) CD8, immature thymocytes and mature CTLs; (h) CD45RO, immature thymocytes and mature memory T cells. (i) MECA-79 immunostaining highlights HEV in the thymic PVS (P). Inset depicts a MECA-79 + HEV at higher magnification. 
al lymphocytes into the PVS.

Our data on the relative proportions of the TES and PVS in normal thymus are consistent with those of Steinmann (2), who used silver staining to identify PVS containing reticular fibers associated with extracellular matrix deposition. The PVS can also be identified by immunostains for fibronectin and laminin $(3,29)$, and by mAb TE7 (11). In this study, we delineated the PVS from the TES (cortex and medulla) using reactivity of the epithelial component of the TES with cytokeratin mAb's as a marker. We have determined the epithelial content of aging thymus, including the development of immunohistologic assays to evaluate the extent of ongoing thymopoiesis in archival FFPE thymus tissues.

We also report a definitive molecular assay for ongoing thymopoiesis in the human thymus, using the LM-PCR assay to detect dsDNA breaks that occur specifically during TCR gene rearrangement. Previous studies have used LM-PCR to investigate V-D-J recombination in mouse thymocytes $(14,22)$. Recent studies have measured the levels of TCR excised circles (TRECs) in blood lymphocytes to estimate levels of thymopoiesis (30-32). However, because TCR excised circles are long-lived in peripheral T cells, and are lost only by dilution during cellular proliferation, a direct correlation between TREC levels and ongoing thymopoiesis cannot be made with this assay. The LM-PCR assay provides a snapshot of ongoing TCR gene rearrangement at the time of tissue harvest, and this provides a definitive measure of thymopoiesis in cases where fresh thymus tissue is available.

Our studies of lymphocytes in the TES demonstrated that the presence of mature CTLs, as indicated by expression of the TIA-1 granule antigen, is rare within the TES. These results fail to directly support the hypothesis of Hartwig and Steinmann (6) that such cells are responsible for induction of atrophy in aging thymus. We did find that TIA- $1^{+}$CTLs are often present in the PVS of both normal and MG adult thymus tissues (Figure 4c). Because cells in the PVS are separated from the TES by a basement membrane (3), the anatomic localization of thymic CTLs make it less likely that these CTLs induce atrophy of the TES through direct effects on thymic epithelial cells. However, CTLs in the PVS could secrete

Table 2

Immunoreactivity of lymphocytes present in the thymic compartments

\begin{tabular}{lccc}
\cline { 2 - 3 } Antibody & Cortex & Medulla & PVS \\
CD3 & ++++ & +++ & +++ \\
CD1a & ++++ & - A & - A \\
mib-1 & +++ & + & ++ \\
CD20 & + & +++ & +++ \\
CD45RA & + & +++ & ++ \\
CD45RO & ++++ & +++ & ++ \\
TIA-1 & - B & - B & + \\
CD4 & ++++ & ++ & ++ \\
CD8 & ++++ & +++ & ++ \\
CD4 + CD8 & ++++ & - & - \\
CD38 & ++++ & +++ & ++ \\
CD3 + CD38 & ++++ & +++ & + \\
\hline
\end{tabular}

Scoring system as described in Methods. Data are representative of 6-70 tissues examined for single stains, and 3-5 tissues examined for double stains. APositive on

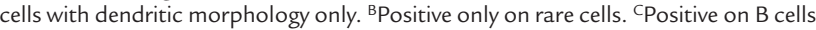
and $T$ cells within germinal centers cytokines that may have direct or indirect effects on the process of thymic atrophy.

We also studied the PVS of MG human thymus tissues in parallel with normal thymus tissue. As reported previously, we found that histologic changes that occur in normal human thymus with aging also occur in MG thymus. The follicular hyperplasia observed in some of the MG thymus tissues is histologically similar to (although quantitatively greater than) changes occurring in normal thymus during aging. Therefore, both normal and MG thymus can be used to illustrate the changes that occur in the PVS and TES of the human thymus with aging.

We report several other novel observations. First, although eosinophils have previously been identified within thymus tissue of infants $(33,34)$, their location within the PVS and their decreased prevalence with age has never been described. Our data showed that thymus from children 2 years of age or younger potentially contains sufficient IL-5 to support eosinophil differentiation, consistent with the hypothesis that eosinophils differentiate in situ within the PVS of pediatric thymus early in life. However, because IL-5 has been shown to also be chemotactic for eosinophils $(35,36)$, increased eosinophil migration into the PVS of IL-5-expressing pediatric thymus tissues cannot be ruled out.

Although several studies have suggested that lymphocytes present within the PVS may be of peripheral origin (see below), our study is the first to demonstrate that these PVS lymphocytes are immunophenotypically consistent with peripheral mature cells. Although many surface markers expressed on PVS lymphocytes are also expressed on lymphocytes present in the thymic medulla, the presence of large numbers of $\mathrm{CD} \mathrm{a}^{-}$,

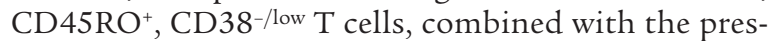
ence of TIA- $1^{+}$-activated CTLs within the PVS, demonstrated that at least some PVS T cells are not new virgin $\mathrm{T}$ cells exiting from the thymic medulla, and therefore most likely migrated from the periphery. The presence of B cells located clearly within the thymic medulla (Figure $4 \mathrm{~b}$ ) raises the question of whether those cells arose in the medulla, in contrast to PVS B cells that may come from the periphery. The contribution of the thymus to the process of B-cell development has not been studied adequately, and the potential contributions of thymic B cells to the peripheral immune B-cell repertoire remain unclear.

The question of whether T cells in the thymic PVS originate within the thymus or migrate from the periphery has been previously addressed in animal models. Savino et al. (29) described the existence of giant thymic PVS in the nonobese diabetic mouse. Cells within the PVS of these animals were resistant to sublethal irradiation with $\mathrm{x}$-rays and hydrocortisone treatment, and were concluded to be mature lymphocytes, consistent with our more extensive phenotypic characterization of PVS cells in human thymus.

Finally, the identification and localization of HEVs within the PVS of human thymus is important. The existence of HEVs within the thymus has previously been reported in preleukemic mice (37), although those authors did not recognize the existence of the PVS and described these HEVs as occurring within the thymic medulla. "Occasion- 
al, predominately medullary MECA-79+ $\mathrm{HEV}[\mathrm{s}]$ " were reported previously in postnatal human thymus (38); however, the significance was not discussed and anatomic location was similarly imprecise. The expression of MECA-79 (a ligand for L-selectin) on the HEVs in the PVS suggests a mechanism by which peripheral lymphocytes could access the thymic PVS, similar to mechanisms described in peripheral lymph nodes and sites of inflammation (38). Our studies using in vitro lymphocyte binding assays demonstrate that peripheral blood lymphocytes can bind to thymic HEVs and thus potentially migrate into the thymic PVS. The function of these PVS lymphocytes in relation to the process of thymic involution has not been established, although temporally, the largest infiltrate volumes are seen in the age ranges where more rapid involution occurs. The mechanisms regulating HEV development in the PVS are still unknown.

Taken together, our studies suggest that the thymic PVS is a compartment of the peripheral immune system that is not directly involved in thymopoiesis. Understanding the relationships between the trafficking of peripheral lymphocytes to thymic PVS should lead to insights into mechanisms of thymic atrophy and control of postnatal thymic function.

\section{Acknowledgments}

This study was supported by National Institutes of Health grants AG-16826 (to L.P. Hale) and CA-28936 (to B.F. Haynes), and by Department of Defense grant DAMD17-97-1-7045 (to K.G. Flores). The authors would like to thank Michelle McMurry and Michael Krangel for discussions and suggestions regarding LM-PCR; James Thomasch and Joseph Horvatinovich for assistance with flow cytometry; and Paula Greer for technical assistance.

1. Steinmann, G.G., Klaus, B, and Muller-Hermelink, H.-K. 1985. The involution of the ageing human thymic epithelium is independent of puberty. A morphometric study. Scand. J. Immunol. 22:563-575.

2. Steinmann, G.G. 1986. Changes in the human thymus during aging. Curr Top. Pathol. 75:43-88.

3. Bofill, M., et al. 1985. Microenvironments in the normal thymus and the thymus in myasthenia gravis. Am. J. Pathol. 119:462-473.

4. Kato, S., and Schoefl, G.I. 1989. Microvasculature of normal and involuted mouse thymus: light- and electro-microscopic study. Acta. Anat. (Basel). 135:1-11.

5. Tamaoki, N., Habu, S., and Kameya, T. 1971. Thymic lymphoid follicles in autoimmune diseases: histological, histochemical, and electron microscopic studies. Keio J. Med. 20:57-68.

6. Hartwig, M., and Steinmann, G. 1994. On a causal mechanism of chronic thymic involution in man. Mech. Ageing Dev. 75:151-156.

7. Krenacs, L., Tiszalvicz, L., Krenacs, T., and Boumsell, L. 1993. Immunohistochemical detection of CD1a antigen in formalin-fixed and paraffinembedded tissue sections with monoclonal antibody O10. J. Pathol. 171:99-104.

8. Mason, D.Y., et al. 1989. Detection of T cells in paraffin wax embedded tissue using antibodies against a peptide sequence from CD3 antigen. J. Clin. Pathol. 42:1194-1200.

9. Key, G., et al. 1993. New Ki-67-equivalent murine monoclonal antibodies (MIB 1-3), generated against bacterially expressed parts of for the Ki-67 DNA containing three 62 base pair repetitive elements encoding the Ki-67 epitope. Lab Invest. 68:629-636.

10. Anderson, P., et al. 1990. A monoclonal antibody reactive with a 15-kDa cytoplasmic granule-associated protein defines a subpopulation of CD8+ Tlym- phocytes. J. Immunol. 144:574-582.

11. Haynes, B.F., Scearce, R.M., Lobach, D.F., and Hensley, L.L. 1984. Phenotypic characterization and ontogeny of mesodermal-derived and endocrine epithelial components of the human thymic microenvironment. J. Exp. Med. 159:1149-1168.

12. Streeter, P.R., Berg, E.L., Rouse, B.T.N., Bargatze, R.F., and Butcher, E.C. 1988. A tissue-specific endothelial cell molecule involved in lymphocyte homing. Nature. 331:41-46.

13. Streeter, P.R., Rouse, B.T.N., and Butcher, E.C. 1988. Immunohistologic and functional characterization of a vascular addressin involved in lymphocyte homing into peripheral lymph nodes. J. Cell Biol. 107:1853-1862.

14. McMurry, M., Hernandez-Martin, C., Lauzurica, P., and Krangel, M.S. 1997. Enhancer control of local accessibility to the VDJ recombinase. Mol. Cell. Biol. 17:4553-4561.

15. Ausubel, F.M., et al. 1997. Current protocols in molecular biology. John Wiley \& Sons. New York, NY. 15.5.10-15.5.19.

16. Toyonaga, B., Yoshikai, Y., Vadasz, V., Chin, B., and Mak, T.W. 1985. Organization and sequences of the diversity, joining, and constant regions of the human T-cell beta chain. Proc. Natl. Acad. Sci. USA. 82:8624-8628.

17. Stamper, H.B., and Woodruff, J.J. 1976. Lymphocyte homing into lymph nodes: in vitro demonstration of the selective affinity of recirculating lymphocytes for high endothelial venules. J. Exp. Med. 144:828-833.

18. Steeber, D.A., Green, N.E., Sato, S., and Tedder, T.F. 1996. Lymphocyte migration in L-selectin-deficient mice: altered subset migration and aging of the immune system. J. Immunol. 157:1096-1106.

19. Rothenberg, E.V. 1992. The development of functionally responsive T cells. Adv. Immunol. 51:85-214.

20. Haynes, B.F., Denning, S.M., Le, P.T., and Singer, K.H. 1990. Human intrathymic T cell differentiation. Semin. Immunol. 2:67-77.

21. Malavasi, F., et al. 1994. Human CD38: a glycoprotein in search of a function. Immunol. Today. 15:95-97.

22. Roth, D.B., Zhu, C., and Gellert, M. 1993. Characterization of broken DNA molecules associated with V(D)J recombination. Proc. Natl. Acad. Sci. USA. 90:10788-10792.

23. Sanderson, C.J., et al. 1998. Interleukin-5. In Cytokines. A. Mire-Sluis and R. Thorpe, editors. Academic Press. London, United Kingdom. 69-80.

24. Dent, L.A., Strath, M., Mellor, A.L., and Sanderson, C.J. 1990. Eosinophilia in transgenic mice expressing IL-5. J. Exp. Med. 172:1425-1431.

25. Gossman, J., Lohler, J., and Lehmann-Grube, F. 1991. Entry of antivirally active $\mathrm{T}$ lymphocytes into the thymus of virus-infected mice. J. Immunol. 146:293-297.

26. Berg, E.L., Robinson, M.K., Warnock, R.A., and Butcher, E.C. 1991. The human peripheral lymph node vascular addressin is a ligand for LECAM-1, the peripheral lymph node homing receptor. J. Cell Biol. 114:343-349.

27. Bargatze, R.F., Jutila, M.A, and Butcher, E.C. 1995. Distinct roles of L-selectin and integrins $\alpha_{4} \beta_{7}$ and LFA-1 in lymphocyte homing to Peyer's patch-HEV in situ: the multi-step model confirmed and refined. Immunity. 3:99-108.

28. Berlin, C., et al. 1993. $\alpha_{4} \beta_{7}$ integrin mediates lymphocyte binding to mucosal vascular addressin MAdCAM-1. Cell. 74:185-195.

29. Savino, W., Carnaud, C., Luan, J.-J., Bach, J.-F., and Dardenne, M. 1993. Characterization of the extracellular matrix-containing giant perivascular spaces in the NOD mouse thymus. Diabetes. 42:134-140.

30. Douek, D.C., et al. 1998. Changes in thymic function with age and during treatment of HIV infection. Nature. 396:690-695.

31. Haynes, B.F., et al. 1999. Analysis of the adult thymus in reconstitution of T lymphocytes in HIV-1 infection. J. Clin. Invest. 103:453-460.

32. Jamieson, B.D., et al. 1999. Generation of functional thymocytes in the human adult. Immunity. 10:569-575.

33. Dourov, N. 1982. L'examen microscopique du thymus au cours de la periode perinatale. Ann. Pathol. 3:255-261.

34. Muller, E. 1977. Localization of eosinophils in the thymus by peroxidase reaction. Histochemistry. 52:273-279.

35. Yamaguchi, Y., Hayashi,Y., Sugama, Y., Miura, Y., and Kasahara, T. 1988. Highly purified murine interleukin-5 (IL-5) stimulates eosinophil function and prolongs in vitro survival. J. Exp. Med. 167:1737-1742.

36. Wang, J.M., et al. 1989. Recombinant human interleukin 5 is a selective eosinophil chemoattractant. Eur. J. Immunol. 19:701-705.

37. Michie, S.A., Streeter, P.R., Butcher, E.C., and Rouse, R.V. 1995. L-selectin and $\alpha_{4} \beta_{7}$ integrin homing receptor pathways mediate peripheral lymphocyte traffic to AKR mouse hyperplastic thymus. Am. J. Pathol. 147:412-421.

38. Michie, S.A., Streeter, P.R., Bolt, P.A., Butcher, E.C., and Picker, L.J. 1993. The human peripheral lymph node vascular addressin: an inducible endothelial antigen involved in lymphocyte homing. Am. J. Pathol. 143:1688-1698. 